\begin{tabular}{lllllllllllllllllllllllllllllllll}
\hline$R$ & $E$ & $V$ & I & S & T & A & D & E & E & S & T & U & D & I & O & S & I & N & T & E & R & N & A & C & I & O & N & A & L & E & S
\end{tabular}

\title{
Evolución de la política exterior de Chile. Oportunidades para la juventud
}

\author{
Fernando Alvarado
}

\begin{abstract}
Una gestión exitosa con resultados brillantes. Con esta frase puede resumirse la política exterior chilena desde comienzos de los años noventa. Chile ha logrado acuerdos de diversa índole con diversos Estados del mundo. Sin embargo, los especialistas y la prensa tienden a analizar estos tratados, ya sea políticos o comerciales, exclusivamente desde el punto de vista comercial y muchas veces no recuerdan que los acuerdos no sólo traen beneficios económicos, sino que los resultados de la política exterior que culmina en estos acuerdos también beneficia a los jóvenes y al resto de la población en la forma de nuevas oportunidades en los ámbitos social y cultural. El objetivo de este artículo es revisar brevemente la política exterior chilena, en especial las relaciones con la Unión Europea, y su vinculación con los jóvenes chilenos y las oportunidades que se presentan a este importante sector de la población, tema sobre el cual la literatura es aún muy escasa. El texto analizará brevemente la evolución de la política exterior de Chile desde comienzos de los años noventa y centrará la atención en las relaciones entre Chile y la Unión Europea, analizando las oportunidades que se han abierto para los jóvenes chilenos y mostrando que la política exterior chilena no sólo gira en torno a temas comerciales y económicos, sino que atribuye prioridad a temas políticos, culturales y sociales.
\end{abstract}

\section{INTRODUCCIÓN}

1 mundo cambió a fines de los años ochenta. El fin de la Guerra Fría, con la caída del muro de Berlín, trajo nuevas prioridades a la política exterior de los diferentes países. Tras dece- nios de vivir en un mundo bipolar, en el cual las relaciones internacionales giraban en torno a temas de seguridad, comenzó una época de intensificación de las relaciones comerciales antes que de la seguridad frente a un eventual conflicto entre las otrora superpotencias. Se inició 
una era de predominio militar de una sola potencia y en que la economía mundial adquiere características cada vez más globales. Los nuevos tiempos se caracterizan por eventos económicos locales, por ejemplo, crisis económicas que repercuten a nivel global, y eventos globales que repercuten en las economías locales. Desde fines de los años ochenta aumenta además el intercambio comercial y se intensifican los flujos financieros a nivel mundial. Al mismo tiempo, se fortalece un sistema de gobierno que en los años setenta y parte de los ochenta estuvo ausente de la mayoría de los países latinoamericanos: la democracia. A fines de los ochenta la democracia pasa a ser el sistema de gobierno de una gran cantidad de países en el mundo, poniendo fin a decenios de gobiernos de facto, tanto de izquierda como de derecha. La democracia es legítima como sistema de gobierno en América Latina, Chile es un caso emblemático. Al respecto, la alta estabilidad política y económica generada desde el retorno a la democracia a comienzos de los noventa y especialmente su influencia en las relaciones exteriores chilenas, han transformado a nuestro país en paradigma de una transición democrática exitosa en un contexto de creciente globalización económica.

\footnotetext{
La política exterior se ha caracterizado por aplicar una estrategia de inserción en la economía internacional mediante tratados comerciales.
}

El presente artículo busca, en primer lugar mostrar que la política exterior chilena se ha caracterizado por aplicar una estrategia de inserción en la economía mundial mediante tratados comerciales con diferentes países del mundo. Se analizan específicamente las relaciones entre Chile y la Unión Europea, que alcanzaron su punto más alto en 2002, cuando el Estado chileno y la Unión Europea acordaron firmar un tratado de libre comercio y cooperación. Sin embargo, la política exterior de nuestro país con Europa y el resto del mundo no se ha caracterizado sólo por los temas comerciales. Analizaremos cómo otros temas de cooperación han permitido a Chile potenciar uno de los sectores más importantes de la sociedad: los jóvenes. Examinaremos cuáles han sido las principales áreas de cooperación que Chile ha logrado implementar gracias a su política exterior en relación con los jóvenes, mediante un trabajo orientado a dar impulso a la política pública de juventud.

\section{Comienzos de los AÑOS NOVENTA: SE INTENSIFICAN LAS RELACIONES COMERCIALES}

A comienzos de los años noventa la política exterior chilena comenzó a aplicar una estrategia de reinserción política y económica en un mundo globalizado. La primera tenía como objetivo dejar de ser el país políticamente excluido durante el régimen militar y condenado en reiteradas ocasiones por violaciones a los derechos humanos; la segunda había co- 
menzado a aplicarse durante el gobierno de Pinochet, y buscaba ser parte de la globalización económica.

\section{El concepto de globalización ha sido divinizado y satanizado.}

$\mathrm{Si}$ analizamos el concepto de globalización, podemos concluir que es uno de los más estudiados de los últimos años, y ha llegado a ser divinizado y satanizado, entendido como útil o inútil para el análisis de los países en vías de desarrollo. Cabe señalar que la opinión sobre la globalización es tan diversa como la calidad de los artículos que lo estudian y no se ha logrado una definición clara, ni menos teorizar sobre el concepto ${ }^{1}$. Si se examina la génesis del concepto de globalización, lo encontramos en los años ochenta como un término más bien periodístico ${ }^{2}$, referido a la difusión de las noticias a través de medios que operan en forma transnacional. Sin embargo, con el paso del tiempo se traslada a las ciencias sociales. Si se parte de la premisa de que la globalización económica se refiere a la apertura de las economías nacionales a una economía mundial y a una interdependencia cada vez mayor entre ellas, se podría concluir que las opiniones sobre la génesis del concepto de globalización, en especial la economía, son diversas y en algunos casos excluyentes. Para Hirst y Thompson, la globalización de los mercados, especialmente en lo referido al comercio exterior, comienza a intensificarse al inicio del siglo XIX y se extiende hasta la Primera Guerra Mundial, momento en que decae, para recuperarse y crecer vertiginosamente desde fines de la Segunda Guerra Mundial hasta nuestros días ${ }^{3}$. Waters tiene una impresión diferente sobre la globalización y su génesis. Si bien coincide con otros autores respecto de la antigüedad del fenómeno, considera que recién desde fines de los ochenta, con el colapso de la Unión Soviética, comienza a definirse el concepto de globalización económica desde un punto de vista académico, lo cual coincidiría con la intensificación del mismo proceso. Es justamente a raíz del fin de la Guerra Fría que se incrementan las relaciones comerciales a nivel mundial. Así, el concepto central de seguridad que postulan la ex Unión soviética y los Estados Unidos pierde valor cuando subsiste una sola potencia.

Para la Comisión Económica para América Latina y el Caribe (CEPAL), el concepto de globalización define el proceso de transformación tecnológica e institucional de la economía mundial contemporánea. Sin embargo, el fenómeno y sus elementos constitutivos no están claramente diferenciados, lo que puede llevar a definir el término de acuerdo con los resultados ${ }^{4}$.

${ }^{1}$ Bodemer, Klaus, "La globalización. Un concepto y sus problemas", en Nueva sociedad, № 156, julio-agosto, Caracas, 1998.

${ }^{2}$ Francia Filho, Marcilio Toscano, "Integración regional y la globalización de la economía; las dos caras del nuevo orden mundial”, en Revista Estudios Públicos, № 100, abril-junio, 1998.

${ }^{3}$ Ver Hirts, Paul y Thompson, Graham, Globalization in Question, Cambridge, 1996.

${ }^{4}$ CEPAL, Panorama de la inserción internacional de América Latina y el Caribe, Santiago, 1996. 
En definitiva, las opiniones de los diferentes autores que figuran en estas líneas esbozan lo complejo que resulta definir el concepto de globalización y especialmente su génesis. En general, las características más comúnmente presentes en la literatura son la apertura comercial, una economía abierta a la libre competencia, la movilidad de bienes, capitales y servicios, la privatización de diversas actividades otrora en manos del Estado, las reformas estructurales neoliberales del Estado, la introducción de reglas, mecanismos e instituciones de gobierno a escala global, que permiten la interacción de unidades locales, nacionales y regionales y llegar a converger en una conducción eficiente a nivel global ${ }^{5}$.

\section{La globalización envuelve a toda sociedad y Estado en un sistema de dependencias recíprocas.}

Sin embargo, estas características de la globalización se relacionan con aspectos económicos, que son importantes pero no únicos. También se asocia la globalización a la difusión de la democracia en el mundo post Guerra Fría como el mejor sistema de gobierno. Como señala el autor alemán Ulrich Beck, es una reproducción y condensación de interacciones que rebasan todo límite, un proceso que envuelve a toda sociedad y Estado en un complejo sistema de dependencias recíprocas ${ }^{6}$.

\section{LOS JÓVENES Y LA GLOBALIZACIÓN}

Los jóvenes son un grupo muy perceptivo de la sociedad. Se podría definir como un sector de la sociedad capaz de captar claramente los procesos sociales de cambio en su ambivalencia de padecer y configurar. Tanto en su vivencia como en sus reacciones, pero también en su padecer, se encuentran los puntos de ruptura de los procesos sociales ${ }^{7}$. Quizás es este grupo de la sociedad el que tiene mayor capacidad para percibir los cambios que se están generando a nivel local y global. Podría concluir que los jóvenes son quienes mejor logran captar una de las características más mencionadas de la globalización, en la cual acontecimientos locales repercuten en el ámbito global y lo global repercute en lo local. Sin embargo, otros autores mencionan la necesidad de distinguir entre el joven globalizador o actor principal en el proceso y el joven globalizado. Según Vásquez Montalban se instala así una nueva dialéctica conflictiva, que es especialmente visible en América Latina, ya que no es lo mismo abordar la globalización desde el punto de vista del globalizador, que asume un rol protagónico en el proceso de cambio, que desde aquel del

\footnotetext{
${ }^{5}$ Gruppe von Lissabon, Grenzen des Wettbewerbs, Colonia, Luchterhand, 1997, p. 151.

${ }^{6}$ Ver Beck, Ulrich, Was ist Globalisierung?, Frankfurt, Suhrkamp Verlag, 1998

${ }^{7}$ Eckholt, Margit/Hünermann, Peter 1998, “Opción por los jóvenes en Latinoamérica en la vorágines de los procesos de globalización”, en: Eckholt, Margit/Hünermann, Peter 1998, La juventud latinoamericana en los procesos de globalización, Buenos Aires; 284 pp.
} 
globalizado, que se podría definir como un sujeto más bien pasivo que asume la globalización sin poder influir en ella ${ }^{8}$. Se transforma así la globalización en una amenaza, pero también en gran oportunidad para los jóvenes.

\section{El sujeto globalizado asume la globalización sin poder influir en ella.}

Si analizamos la globalización desde una perspectiva social se comprueba que ha producido diversas reacciones en las distintas sociedades, tanto en países desarrollados como en vías de desarrollo. En Francia, los agricultores protestan por la apertura de los mercados agrícolas en especial a países exportadores que pertenecen al Tercer Mundo, mientras que en Seattle o en Québec, diversos grupos del tercer sector protestan por las reuniones de la Organización Mundial de Comercio, entidad símbolo de la globalización económica, por promover la apertura comercial de los países miembros.

Ahora bien, en los países de América Latina la juventud asocia la globalización a una exigencia cada vez mayor en sus vidas. Se genera por una parte la percepción de que hay oportunidades para los jóvenes, de que se abren espacios de desarrollo personal a través de la interrelación cada vez más intensa con otros países en diversos ámbitos. Sin embargo, se ha podido constatar que el principal desafío que enfrentan las nuevas generaciones es la escasa y defectuosa capacitación de muchas de ellas pertenecientes a estratos bajos y especialmente medios, que si bien han podido permanecer más tiempo en el sistema educativo formal, al tratar de incorporarse al mercado de trabajo tropiezan con dificultades por falta de "experiencia" y capacitación. Los que sí tienen esa capacitación o profesionalización no se incorporan necesariamente al mercado laboral porque encuentran una elevada "selectividad"9 en los requerimientos laborales. En definitiva, los jóvenes asumen la globalización como una gran exigencia, pero también como una oportunidad real de crecimiento y desarrollo personal.

A continuación, se examinarán brevemente las relaciones de Chile con la Unión Europea en la década de los noventa, para finalizar con una revisión del acuerdo de cooperación de 2002 a fin de destacar las oportunidades que existían para los jóvenes antes del tratado y las que se les abren ahora.

\section{Política exterior de Chile desde COMIENZOS DEL DECENIO DE 1990:}

RELACIONES POLÍTICAS Y ECONÓMICAS CON LA UNIÓN EUROPEA

El retorno de Chile a un régimen democrático se formaliza en 1990 con la

\footnotetext{
${ }^{8}$ Vásquez Montalban, M. “Jóvenes globalizados o globalizados”, en Voces, Revista Iberoamericana de Juventud, $\mathrm{N}^{\circ} 1$, nov. 2000, pp. 6.

${ }^{9}$ Rodríguez, Ernesto, "Los jóvenes latinoamericanos: heterogeneidad y diversidad en materia de riesgos, oportunidades y desafíos en la antesala de un nuevo milenio", en Eckholt, Margit/Hünermann, Peter 1998, La juventud latinoamericana en los procesos de globalización, Buenos Aires, pp. 19-50.
} 
primera elección democrática presidencial y parlamentaria. Dentro de este contexto de redemocratización la política exterior chilena adquiere nuevas características. Según van Klaveren, éstas estarían determinadas por cuatro factores ${ }^{10}$ : en primer lugar, la recuperación de las tradiciones de la política exterior de Chile, que fueron suspendidas durante el gobierno militar, y que se reflejó en la mejora de las relaciones con los países vecinos, permitiendo superar en gran medida los problemas limítrofes con ellos, en especial con Argentina. Segundo, la apertura económica iniciada durante el gobierno militar. Sin embargo, esta apertura económica se diferenció por la preocupación de los gobiernos democráticos por tratar de reducir la deuda social heredada del gobierno castrense ${ }^{11}$. Tercero, el retorno de la democracia a Chile, tras casi 17 años de régimen militar. Finalmente la transformación política y económica internacional.

Lo que en definitiva terminó con el predominio de la seguridad mundial en torno a un mundo bipolar fue el fin de la Guerra Fría, que dio paso a un orden dominado por las relaciones económicas ${ }^{12}$. El segundo factor, la continuación de la apertura económica, es a nuestro parecer consecuencia lógica de las transformaciones políticas y económicas de la pos Guerra Fría. Las autoridades políticas y económicas del primer gobierno de la Concertación vieron la necesidad de continuar con una economía abierta al mundo, en que las exportaciones juegan un papel cada vez más importante para un país pequeño como Chile, que depende de su comercio exterior.

\section{El fin de la Guerra Fría cedió paso a un orden dominado por las relaciones económicas.}

A continuación, se examinarán brevemente las relaciones políticas y económicas de Chile con la Unión Europea desde 1990, esto es, desde la normalización de las relaciones al acuerdo de libre comercio, concluyendo con los espacios y oportunidades abiertas a los jóvenes de Chile, en especial gracias al tratado de cooperación entre el Estado chileno y la Unión.

${ }^{10}$ Van Klaveren, Alberto, "Inserción internacional de Chile”, en Toloza, Cristián/Lahera, Eugenio (eds.), Chile en los noventa, Santiago, Dolmen,, 1998; 117 pp.

${ }^{11}$ La política económica del presidente Patricio Aylwin, presidente del primer gobierno democrático de la era post-autoritaria, se definía con el lema "crecimiento con equidad", que indicaba que se mantenía el modelo económico neoliberal, pero con reformas sociales que buscaban legitimarlo en la población, ya que estaba muy desprestigiado debido a los altos costos sociales que había significado su instauración. Véase, Munck Gerardo, "Authoritarianism, modernization, and democracy in Chile", en Latin American Research Review, Vol. 29, № 2, p. 207; Bresser, Luiz/Maravall, José/Przeworski, Adam, Economic reforms in new democracies, Cambridge; Weyland, Kurt, "'Growth with equity', in Chile's new democracy”, en Latin American Research Review, Vol. 32, № 1, 1997.

${ }^{12}$ Rojas Aravena, Francisco, “Chile: cambio político e inserción internacional 1964-1997”, en Revista de Estudios Internacionales, Año XXX, № 118, abril-mayo de 1997. 
Relaciones entre Chile y la Unión EUROPEA DESDE LOS AÑOS NOVENTA

Las relaciones diplomáticas, especialmente económicas, entre Chile y la Unión Europea han sido históricamente muy dinámicas. Con los países europeos Chile ha mantenido fuertes vínculos a lo largo de su historia, en ámbitos tan variados como los políticos, económicos y culturales. Las relaciones económicas son una de las áreas más relevantes en esta relación debido a la gran importancia del mercado europeo, primero como destino de aproximadamente un $30 \%$ de las exportaciones totales, y segundo, por la elevada inversión europea recibida en especial en los años noventa. Cabe señalar que las relaciones económicas y particularmente las políticas se vieron seriamente afectadas durante los años del gobierno militar, situación que cambió con el retorno de nuestro país a la democracia.

\section{El gobierno militar inició una estrategia de apertura comercial pero lo que cambió la imagen de Chile fue el regreso a la democracia.}

Pese a que el gobierno militar inició una estrategia de apertura comercial unilateral para reducir el aislamiento inter- nacional, lo que generó un cambio en la imagen de Chile y le permitió retornar al contacto político con la mayoría de los países del mundo fue la vuelta a la democracia. Claro ejemplo de ello es el giro radical de la relación con los países de la Unión Europea. El hecho de que países como Alemania y Suecia, entre otros, hubiesen acogido a miles de exiliados chilenos durante el gobierno castrense, y de que los países europeos fueran los que más ayudaron a la oposición chilena a organizarse en pos del retorno a la democracia, explica la rápida normalización de las relaciones de los países europeos al iniciarse el gobierno de Aylwin. Con los países de la Unión Europea se produjo un interesante incremento en el intercambio comercial, a la par que países como Inglaterra y España ${ }^{13}$ realizaron fuertes inversiones en nuestro país, relación comercial que en el 2002 se coronó con la firma de un tratado de libre comercio y de cooperación ${ }^{14}$.

\section{LA NUEVA RELACIÓN ECONÓMICA \\ BILATERAL ENTRE ChILE Y LA UNiÓN EUROPEA TRAS EL RETORNO A LA DEMOCRACIA}

A comienzos de los años noventa se inicia una era en que la democracia pasa

${ }^{13}$ En 1990 España invirtió 7537 millones de dólares mientras que la inversión de Inglaterra ascendió a 1402 millones de dólares, PROCHILE, "Estadísticas macroeconómicas", Santiago 2000.

${ }^{14}$ Este párrafo se basa en el artículo del autor "Democracia y relaciones exteriores chilenas en los años '90: factor determinante en la inserción de una economía globalizada”, en Pressacco, Fabián, Democracia en el Mercosur, Instituciones y cultura Política, Universidad Alberto Hurtado y Organización de los Estados Americanos (OEA), Santiago, 2002. 
a ser el sistema de gobierno de un gran número de países del mundo, finalizando así décadas de gobierno de facto, tanto de izquierda como de derecha. La democracia se legitima como el sistema de gobierno en América Latina. Chile es un caso emblemático. La alta estabilidad política y económica generada desde el retorno a la democracia y en especial la influencia de la democracia en las relaciones exteriores chilenas han transformado al país en paradigma de una transición democrática exitosa en el contexto de una creciente globalización económica ${ }^{15}$. Esta nueva realidad influye positivamente en las relaciones de Chile con la Unión Europea desde comienzos de los años noventa.

\section{La estabilidad política y económica ha transformado al país en paradigma de transición democrática exitosa.}

NoRMalizaCión DE LAS RELACIONES Chile-Unión Europea. Hechos ReleVANTES DEL PRIMER GOBIERNO DE LA CONCERTACIÓN

Durante su mandato, el ex presidente Aylwin realizó numerosas giras a diferen- tes lugares del mundo, demostrando así que Chile se reinsertaba en la comunidad internacional. Las visitas presidenciales abarcaron Asia, Oceanía, América Latina, Estados Unidos y Europa. Sin embargo, las relaciones con el viejo continente tuvieron un lugar central en la estrategia de reinserción chilena en la comunidad internacional. La política de intercambio de visitas oficiales hasta los más altos niveles ha servido los propósitos de comunicación y legitimación del gobierno, realzando el contraste entre la nueva posición externa del país y la débil situación política del gobierno militar anterior a Aylwin ${ }^{16}$. En el plano de la cooperación también hubo avances importantes a nivel nacional y comunitario, tanto mediante la ayuda prestada desde la sede de la Comunidad Económica Europea y de los principales países europeos a los programas sociales como mediante el suministro de recursos o donaciones en especie y en asistencia técnica.

Los puntos más importantes expuestos por Aylwin en Europa fueron los señalados en su programa de gobierno, en el cual se hace hincapié en que para que el comercio internacional de Chile sea motor de desarrollo es esencial que las exportaciones tengan amplio acceso a los mercados externos. Cabe destacar que la buena acogida que obtuvo el presidente en países de la Comunidad Económica Europea fue claro reflejo de la disposi-

${ }^{15}$ Alvarado, Fernando 2002, "Democracia y relaciones exteriores chilenas en los años 90: Factor determinante en la inserción de una economía globalizada”, en Pressacco, Fabián 2002, Democracia en el Mercosur, Instituciones y cultura Política, Santiago, Universidad Alberto Hurtado y Organización de los Estados Americanos (OEA), 2002.

${ }^{16}$ Wilhelmy, Manfred/Infante, María Teresa 1993, "La política exterior chilena en los años '90: el gobierno del presidente Aylwin y algunas proyecciones", en Estudios Sociales, № 75, p. 97. 
ción de los líderes europeos a volver a estrechar los contactos con nuestro país. Aunque durante el gobierno militar Europa no disminuyó de manera importante las importaciones de productos chilenos, la inversión del viejo continente en nuestro país fue muy reducida.

\section{La reinserción en la Comunidad Económica Europea fue un proceso trascendente de la política exterior del gobierno de la transición.}

En diciembre de 1990 se dio un paso importante en las relaciones entre Chile y la Comunidad Económica Europea al suscribirse el acuerdo de cooperación llamado de "tercera generación". El acuerdo se inspiró en los principios de cooperación y fue clave para Chile y su desarrollo. Su firma constituyó un reconocimiento de que la Comunidad Europea tiene alguna responsabilidad en la situación de menor desarrollo y pobreza que enfrentaba y enfrenta Chile y en el acuerdo se comprometió solidariamente a apoyar los esfuerzos nacionales de desarrollo económico, social y político poniendo a disposición del país recursos financieros, tecnológicos y culturales ${ }^{17}$ y fue un paso importante en las relaciones entre ambos que culminaron con el acuerdo de libre comercio y cooperación de 2002.

En definitiva, la reinserción de Chile en la Comunidad Económica Europea fue un proceso trascendente en la política exterior del gobierno de la transición en que se lograron importantes acuerdos para determinadas exportaciones chilenas, como las hortofrutícolas, que históricamente se han visto afectadas por las medidas proteccionistas que aplica el bloque europeo a este tipo de productos. El problema pretende superarse en alguna medida gracias al acuerdo de libre comercio que debe ser ratificado por Chile y los países de la Unión Europea.

\section{Profundización de Las Relaciones eConómicas de Chile con la Unión Europea: Negociaciones Para UN \\ TRATADO DE LIBRE COMERCIO Y COOPERACIÓN}

En los años noventa, los presidentes Aylwin y Frei y luego el presidente Ricardo Lagos realizaron diversas visitas oficiales a países de la Unión Europea con el objetivo principal de lograr que las exportaciones chilenas accedieran al mercado del bloque, fomentar la inversión europea en nuestro país ${ }^{18} y$, en definitiva, llegar a un acuerdo de libre comercio con la Unión Europea para eliminar las enormes trabas a que han estado sujetos los productos chilenos en el último decenio.

Chile exporta al mercado europeo fundamentalmente productos primarios y de bajo valor agregado, en especial minerales y productos agrícolas ${ }^{19}$. Estos úl-

${ }^{17}$ Para un resumen de los principales hechos de la política exterior chilena a comienzos de los noventa ver: FLACSO, De la inserción a los acuerdos. La política exterior chilena en 1991, Santiago, 1991.

${ }^{18}$ Ibid.

${ }^{19}$ PROCHILE, Estadísticas Macroeconómicas, Santiago, 1999 
timos son los que se han visto más perjudicados por el proteccionismo de los países de la Unión Europea. Una forma eficiente de obtener ventajas para el ingreso de sus productos a un mercado tan importante como el europeo era negociar un tratado de libre comercio.

\section{Más que el mercado, a los países del viejo continente les interesa usar a Chile de plataforma para operar en Sudamérica.}

En 1996, durante el gobierno de Eduardo Frei Ruiz-Tagle, Chile alcanzó un compromiso con la Unión Europea para negociar un tratado de libre comercio $^{20}$, destino de cerca de un $30 \%$ de las exportaciones chilenas (las exportaciones de la UE a Chile no pasan de un $0,3 \%$ del (otal) ${ }^{21}$. Ahora bien, a los países del viejo continente no les interesa tanto el mercado chileno sino usar a Chile como plataforma para operar en Sudamérica, aprovechando para eso la gran estabilidad política y económica del país. Además, cabe señalar que empresas europeas establecidas en países del Mercosur, en es- pecial Brasil, ven a Chile como puente hacia mercados asiáticos ${ }^{22}$. Es importante anotar que en un comienzo la Unión Europea proponía negociar un acuerdo de libre comercio al mismo tiempo con Chile y con Mercosur.

Con el paso del tiempo, los negociadores chilenos lograron convencer a los europeos de la apertura de la economía chilena, que permitiría una negociación más rápida que con los países del Mercosur que, por ejemplo, aplican aranceles superiores a los chilenos en diversas áreas. Otra variable que impidió la formula de negociar en forma conjunta fue el tema agrícola, especialmente en el caso de los enormes volúmenes de producción de bienes agropecuarios de países como Brasil o Argentina, a los que les resulta más difícil entrar al mercado europeo que a la producción chilena, que es mucho más reducida ${ }^{23}$.

En un viaje a Francia, España y Alemania en junio del año 2001, el presidente Ricardo Lagos fue recibido por el Canciller alemán Gerhard Schroeder, quien le expresó el apoyo de su gobierno, el país más grande y económicamente más fuerte de la Unión Europea, para alcanzar un acuerdo entre la Unión y Chile ${ }^{24}$. La es-

${ }^{20}$ Acuerdo Marco de Cooperación destinado a preparar la asociación política y económica entre Chile y la Comunidad Europea y sus Estados miembros de 1996. DIRECON 2000, "Relaciones económicas internacionales de Chile". Para más antecedentes del proceso de negociación, ver Fernández Amunategui, Mariano, "Estado actual entre Chile y la Unión Europea", CELARE, Relaciones con la Unión Europea: una visión latinoamericana, Santiago, 1995.

${ }^{21}$ Insulza, José Miguel, Ensayos sobre la política exterior de Chile, Santiago, Editorial Los Andes, 1998.

${ }^{22}$ Van Klaveren, Alberto, Inserción internacional de Chile, op. cit.

${ }^{23}$ Entrevista con Alejandro Foxley, marzo 2000, Santiago.

24 "Creemos que debe regir el principio de que el que avance más rápido debe sacar provecho de ello. Nuestra impresión es -y lo respaldo expresamente- que se pueden hacer progresos muy rápida- 
trategia de negociación tripartita, llamada "estrategia puente"por el Comisario de la Unión Europea, Manuel Marín, permite así una negociación separada con Chile.

Después de largas negociaciones entre Chile y la Unión Europea, en el primer semestre de 2002, se establece un acuerdo de libre comercio, que debe ser ratificado por el parlamento chileno y por los 15 países miembros de la Unión Europea. Finaliza así uno de los procesos de negociación mas exitosos realizados por Chile, que implica un gran desafío en las enormes áreas que implica un acuerdo de esta magnitud para el futuro de las relaciones bilaterales de Chile con los 15 países de la Unión.

\section{LOS JÓVENES CHILENOS COMO} BENEFICIARIOS DE LOS ACUERDOS

INTERNACIONALES: DE QUÉ JÓVENES ESTAMOS HABLANDO

Cuando se habla de los jóvenes chilenos y su vinculación con la política exterior del país, es importante examinar previamente sus características principales, sus valores, sus sueños y, en general, su visión de futuro. El estudio más importante sobre la juventud chilena es la Tercera Encuesta Nacional de Juventud, investigación llevada a cabo por el Instituto Nacional de la Juventud. Si bien no hay estudios sobre la percepción de los jóvenes acerca de las relaciones internacionales, veremos cuáles son sus tendencias de opinión y cómo influyen las oportunidades que les abre la política exterior chilena, ya sea a través de los tratados de libre comercio o, de manera especial, por los tratados de cooperación.

\section{Los factores más importantes para tener éxito en la vida son la constancia y el espíritu de trabajo.}

Para los jóvenes chilenos, el factor principal para tener éxito en la vida es la constancia y el espíritu de trabajo (58\%), seguido por una buena educación (31\%) y metas claras (28\%). Es importante señalar que el hecho de tener contactos, lo que popularmente se llama tener "pitutos" para acceder a beneficios laborales o de otro tipo en la vida sólo ocupa el noveno lugar en las apreciaciones de los jóvenes, con sólo un $9 \%$.

Según los propios jóvenes, la juventud es una etapa de grandes decisiones (45\%) y un $28 \%$ piensa que es para aprender cosas que sirven para tener éxito. Sólo un $9 \%$ declara que lo principal es pasarlo bien $^{26}$. Para este grupo de la sociedad chilena, lo más importante en la vida para ser feliz es desarrollarse como persona, tener un buen trabajo o profesión y cons-

mente en las negociaciones entre la Unión Europea y Chile. Las relaciones políticas no podrían ser mejores. Chile en estos momentos está en una fase de desarrollo muy buena”. Declaraciones del Canciller alemán después de una conversación privada con el Presidente chileno Ricardo Lagos en Berlín el 1 junio de 2001. Biblioteca del Congreso Nacional, resumen semanal online, <www.bcn.cl>.

${ }^{25}$ INJUV, Tercera Encuesta Nacional de la Juventud 2002, Santiago, 105.

${ }^{26}$ INJUV, Tercera Encuesta Nacional de la Juventud 2002, Santiago, 95. 
truir una buena familia. Cabe señalar que los jóvenes opinan que la educación y el trabajo son los principales medios para lograr la inclusión social ${ }^{27}$.

En cuanto al futuro, se puede concluir que los jóvenes chilenos lo ven con mucho optimismo. Más del $95 \%$ piensa que dentro de 5 años estarán un poco mejor o mucho mejor.

\section{La educación y el trabajo son los principales medios para lograr la inclusión social.}

En cuanto a su percepción de la política, datos empíricos sobre el tema juventud muestran que sólo un $13 \%$ del total de personas inscritas en los registros electorales son jóvenes. A esto hay que agregar un dato aún más preocupante: aproximadamente la mitad de los jóvenes encuestados por un estudio realizado por el Instituto Nacional de la Juventud (INJUV) afirma que la democracia es un sistema de gobierno como cualquier otro y sólo un $48,8 \%$ piensa que es el mejor. A modo de referencia, en España 79\% de la juventud considera la democracia como el mejor sistema de gobierno. A lo anterior pueden agregarse los siguientes datos:

La confianza de los jóvenes en los actores políticos es, en general, muy baja. El presidente de la República, como institución, es el actor político mejor evaluado. Un $20 \%$ de los jóvenes tiene mucha confianza a esta institución; $48 \%$ tiene poca confianza y $31 \%$ manifiesta una total desconfianza. En el caso de los senadores y diputados, sólo el 3\% de los jóvenes expresó tener mucha confianza en estos actores políticos. El 46,5\% les tiene poca confianza y un $50,5 \%$ de los jóvenes encuestados señaló tener total desconfianza en esta institución política. Aún peor evaluados son los partidos políticos, con casi un $60 \%$ de desconfianza. Lapidario es también el grado de interés en participar en un partido político, donde el $88,7 \%$ de los encuestados señala su falta de interés por participar en ellos.

Estos indicadores reflejan un notorio desinterés de los jóvenes por la política. Esto es visible en el alejamiento gradual de los jóvenes de la política contingente y el escaso volumen de votantes de este sector.

Sin embargo, el tema joven y su participación en política no debería limitarse a un aspecto electoral. Al evaluar a los jóvenes y su relación con la política deberían tenerse presentes dos aspectos: en primer lugar, la falta de interés en la política y la escasa participación electoral. Desde las elecciones presidenciales, parlamentarias y municipales de 1998 a la fecha, hay una clara tendencia a la disminución del número de electores, que obedece a la baja paulatina de la participación de los jóvenes en los actos electorales. De persistir esta tendencia, el número de electores seguirá reduciéndose.

Al parecer, las causas del desinterés de los jóvenes por inscribirse en los registros electorales son diversas. Algunos lo han atribuido a razones de comodidad.

${ }^{27}$ INJUV 2002, Tercera Encuesta Nacional de la Juventud 2002, Santiago, 105. 
Sin embargo, más importante es la convicción de los jóvenes de que con su voto no influyen mayormente en decisiones que ya han sido adoptadas por los partidos, lo que algunos cientistas políticos y sociólogos explican por el sistema electoral binominal existente en Chile, que de alguna manera "impone" un candidato por cada coalición.

\section{La escasa capacidad de influir en la opinión y acción de los partidos políticos ha producido desencanto.}

Sea cual sea la causa del alejamiento de los jóvenes de las urnas, su ausencia y la consiguiente reducción del número de electores inscritos podría llegar a generar en los próximos años una pérdida gradual de la legitimidad de los actos electorales y de las instituciones políticas que renuevan sus miembros mediante el sufragio popular, en especial el Congreso.

Ahora bien, nadie puede afirmar que a los jóvenes no les interese la cosa pública: basta recordar su capacidad de movilización en temas como el pase escolar o manifestaciones de otra índole, como las relacionadas con la protección del medio ambiente. Sin embargo, los partidos políticos que han perdido gradualmente legitimidad sólo están encauzando parcialmente la capacidad de movilización de los jóvenes y sus posibilidades de influir en los temas que les afectan por conducto de organizaciones. Al parecer, hay un desencanto por la escasa capacidad de influir con su opinión y acción en los partidos políticos.
En definitiva, puede concluirse que para los jóvenes, tanto la educación como la constancia y el espíritu de trabajo son los factores determinantes que les permitirán tener éxito en la vida. Los jóvenes tienen fe en sus capacidades, pero no participan en lo público a través de los partidos políticos sino del voluntariado, intercambios, becas de estudio, temas relacionados con el medio ambiente, expresiones culturales y otras formas. A continuación mostraremos cómo las relaciones internacionales, especialmente con Europa, pueden potenciar y estimular estos factores tan importantes.

\section{La Organización Iberoamericana DE JUVENTUD - OIJ}

La Organización Iberoamericana de Juventud - OIJ es un organismo internacional de carácter intergubernamental, creado para promover el diálogo, la concertación y la cooperación en materia de juventud entre los países de Iberoamérica. La sede de este organismo internacional está en Madrid.

La génesis de la OIJ se remonta a 1985, ocasión en que las Naciones Unidas proclamaron el Año Internacional de la Juventud, iniciativa que generó múltiples actividades y acuerdos de carácter mundial centrados en los jóvenes. La OIJ, que se constituyó oficialmente en 1992, es quizás la más importante de ellas.

La OIJ está integrada por las 21 instituciones oficiales responsables de políticas de juventud de Argentina, Bolivia, Brasil, Colombia, Cuba, Costa Rica, Chi- 
le, Ecuador, El Salvador, España, Guatemala, Honduras, México, Nicaragua, Panamá, Paraguay, Perú, Portugal, República Dominicana, Uruguay y Venezuela. Actualmente, la sede de la Organización está en Madrid $^{28}$. Este organismo vincula a instituciones oficiales de gobierno del continente americano y de Europa.

\section{La política exterior también prioriza temas que apuntan a potenciar la inclusión social de los jóvenes.}

Desde 1987, las entidades responsables de asuntos de juventud de los países participantes de la OIJ se han reunido regularmente en once conferencias intergubernamentales sobre políticas de juventud, en las sedes y fechas que se señalan a continuación: Madrid, 1987; Buenos Aires, 1988; San José de Costa Rica, 1989; Quito, Ecuador, 1990; Santiago de Chile, 1991; Sevilla, España, 1992; Punta del Este, Uruguay, 1994; Buenos Aires, 1996; Lisboa, Portugal, 1998; Ciudad de Panamá, 2000 y Salamanca, España, 2002.

Es importante mencionar que Chile fue elegido presidente del consejo directivo de la OIJ en noviembre de 2002 por la unanimidad de los 21 países integrantes, lo que significa un claro reconoci- miento de los países de Iberoamérica a las políticas públicas de juventud de Chile. El cargo lo ejerce Eugenio Ravinet, Director Nacional del Instituto Nacional de la Juventud de Chile hasta noviembre de 2004.

El parlamento chileno ratificó en agosto pasado la adhesión de Chile a la OIJ. Este hecho revela que la política exterior chilena no sólo gira en torno a temas comerciales y económicos, sino que también prioriza temas políticos, culturales y sociales, especialmente cuando apuntan a potenciar la inclusión social de los jóvenes chilenos. Este reconocimiento se ha manifestado en la solicitud oficial de cooperación técnica del INJUV formulada por diversos países de América Latina para sus organismos oficiales de juventud ${ }^{29}$. Objetivos primordiales de la OIJ, como potenciar el tema del voluntariado, el Observatorio de Juventud, Informe Iberoamericano de Juventud (trabajo conjunto con la CEPAL), la carta Iberoamericano de derechos juveniles, entre otras iniciativas, tiene como objetivo hacer más eficiente la focalización de las políticas públicas de juventud y crear las condiciones necesarias para implementar proyectos de fomento de la alfabetización digital para los jóvenes de Iberoamérica.

En medio de la actual crisis de

\footnotetext{
${ }^{28}$ Ver $<$ www.oij.org $>$.

${ }^{29}$ La Agencia de Cooperación Internacional de Chile (AGCI), en el marco del Programa de Asociación Chile- Japón (JCPP), en conjunto con la Dirección de Cooperación Técnica del Ministerio de Relaciones Exteriores y Culto de Costa Rica, organizaron el Seminario Regional "Juventud, Gobernabilidad y Democracia”, que se llevó a cabo durante el 23 al 27 de junio de 2003, en San José de Costa Rica. El INJUV chileno expuso su política pública de juventud, la cual llevó a la petición formal de cooperación técnica a Chile en materias de juventud por países de América Latina.
} 
gobernabilidad de diversos países latinoamericanos, comprometerse con la labor de una organización como la OIJ, que trabaja por la juventud a través de la mejora y la potenciación de políticas públicas adecuadas a su realidad, es un mensaje de apoyo a la participación de los jóvenes en sus países (participación política, comunitaria, ciudadana, etc.) y que genera vínculos entre las sociedades civiles e interés en conocer las experiencias de otros países de la región.

El TRATADO DE COOPERACIÓN ENTRE Chile y la Unión Europea: OPORTUNIDAD PARA LOS JÓVENES

La cooperación en temas de juventud de los países europeos con Chile ha estado presente en los últimos dos años. Cabe mencionar, entre otros, los proyectos con Inglaterra a través del British Council, que fomentan la inclusión social de los jóve$n^{30}{ }^{30}$, la cooperación con Suecia (Instituto de la Juventud de Suecia), Alemania (Ministerio de Cooperación alemán y la Gesellschaft für Technische Zuzamenarbeits o GTZ), que se desarrollan en especial en forma conjunta con el Instituto Nacional de la Juventud y con corporaciones y organizaciones no gubernamentales cuyo campo de trabajo es la juventud.

El acuerdo entre Chile y la Unión Europea no sólo significará un importante incremento del intercambio comercial.
También abre enormes posibilidades de cooperación entre las dos partes, diversificando los ámbitos de cooperación y profundizando las áreas ya existentes.

\section{Los programas de intercambio han generado vínculos permanentes de amistad entre las nuevas generaciones.}

Entre las posibilidades que ofrece el tratado de cooperación a los jóvenes, cabe mencionar las siguientes:

Los programas de intercambio para jóvenes y de voluntariado son una oportunidad real de cooperación. En el viejo continente se han desarrollado diversos programas de intercambio entre jóvenes de diversos países, especialmente a través del programa YOUTH de la Unión Europea. Se abren espacios de cooperación en temas de juventud como programas de intercambio entre jóvenes chilenos y europeos, y se fomenta el voluntariado y otras formas de participación juvenil. El acuerdo abre la posibilidad de desarrollar lazos de cooperación entre las instituciones oficiales de la juventud de Chile y de Europa, lo que se contempla en el Título IV, Artículo 41 y 42 del acuerdo, que prevé la cooperación en materia de administración pública y cooperación interinstitucional. Ejemplo de ello es la invitación a participar en un proyecto de intercambio de jóvenes y voluntariado del YOUTH PROGRAM, financiado por una ins-

\footnotetext{
${ }^{30}<$ http://www.britishcouncil.cl/spanish/governance/chldreamsteams.htm>. $<\mathrm{http}$ ://www2.britishcouncil.org/dreamsandteams-about.htm $>$.
} 
titución española, al que se sumarán otros países europeos y latinoamericanos. Se generan así vínculos permanentes de amistad entre las nuevas generaciones de los países participantes, acercando a las culturas y fomentando la tolerancia, y el respeto y ayudando a la educación por la paz. Es importante mencionar que el INJUV y la embajada de la Unión Europea en Chile han establecido un convenio de colaboración en virtud del cual el organismo estatal difundirá por conducto de sus direcciones regionales y de sus cincuenta centros de información regionales lo largo de Chile las oportunidades que ofrece a los jóvenes el tratado de cooperación.

\section{Las becas son otra gran oportunidad en las relaciones de Chile y Europa.}

Las becas son otra gran oportunidad en las relaciones de Chile y Europa. En especial las becas Alban, cuyo punto focal es la Universidad de Chile, permiten a los jóvenes chilenos realizar estudios de postgrado en universidades europeas. Este proyecto ha sido muy exitoso y ha permitido a jóvenes chilenos y de otros países de América Latina acceder a becas de hasta 2 años para personas de hasta 45 años de edad, a fin de que realicen estudios de postgrado, tanto de magíster como de doctorado.

Sería muy positivo poder ampliar estos programas a otros países de América Latina y, a nuestro juicio, éste es uno de los desafíos que debería plantearse en la III Cumbre de Presidentes de América
Latina y Europa, que tendrá lugar en Guadalajara en mayo de 2004. La iniciativa será presentada por el Instituto $\mathrm{Na}$ cional de la Juventud de Chile como presidente de la Organización Iberoamericana de Juventud y por México como vicepresidente de dicha organización intergubernamental. El alero que brinda la OIJ a acuerdos entre los países de América Latina y Europa es muy importante para potenciar las relaciones entre los jóvenes de ambas regiones del mundo.

Por último, el acuerdo menciona la posibilidad de que Chile pueda acceder a los programas de cooperación de la Unión Europea para sus Estados miembros en el marco de la relación de asociación o "upgrading". Esta modalidad de cooperación abre interesantes oportunidades de cooperación con la Unión Europea y permite que Chile participe en programas europeos existentes mediante una relación de financiamiento conjunto entre ambas partes. La propuesta de Chile se debe formular al Consejo de Asociación, entidad rectora del Acuerdo con la Unión Europea, ya que esta institución puede apoyar esta clase de iniciativas formulando recomendaciones para que se lleve a cabo la propuesta chilena. Uno de los proyectos que se podría implementar en Chile y que cabe en esta categoría es el programa ERASMUS, que permite a los estudiantes universitarios de países europeos permanecer algunos semestres de su carrera en una universidad de otro país de la UE.

En definitiva, las relaciones entre los jóvenes de Chile y los países Europeos se verán incrementadas gracias al acuerdo 
de cooperación. Son especialmente las do aún más a los jóvenes a vislumbrar las áreas de voluntariado, intercambios, be- relaciones exteriores de Chile con otros cas, y proyectos culturales las que son las Estados como una oportunidad real para más favorecidas por el acuerdo, acercan- su desarrollo como ciudadano. 